\title{
Facial Onset Sensory and Motor Neuropathy Presented with Bilateral Neurotrophic Keratopathy
}

\author{
Maryam Naser MD ${ }^{1 *}$, Amy Patel MD², Guy Massry MD ${ }^{2}$ and Nafiseh Hashemi MD ${ }^{1}$ \\ ${ }^{1}$ Department of Neuro-ophthalmology Clinic, Hashemi Eye Care, USA \\ ${ }^{2}$ Cedars Sinai Medical Center, USA
}

*Corresponding author: Maryam Naser, Neuro-ophthalmology Clinic, Hashemi Eye

Care, Encino, California, USA.

\section{Abstract}

We report a rare case of Facial Onset Sensory and Motor Neuropathy (FOSMN) which was diagnosed with superior ophthalmic nerve biopsy. Facial pain and paresthesia improved after treatment with Intravenous Immunoglobulin (IVIG).

\section{Case}

An 83-year-old female with a history of hypertension, coronary artery disease, and hyperlipidemia presented with ocular and trigeminal region pain initially presented to an outside general physician for right sided frontal pain, facial weakness and eye pain that started on one side and subsequently progressed to the contralateral side. Upon initial ophthalmologic examination, she was noted to have trigeminal neuropathy with loss of sensation in the v1, v2 and v3 distribution bilaterally. In addition, she was noted to have partial facial nerve paralysis with orbicularis weakness, difficulty with speech, hoarseness and difficulty swallowing. Her visual acuity was significantly reduced to count finger bilaterally due to corneal scarring and epithelial defects were noted. Given extensive findings of involvement of cranial nerve 5th and 7th, a workup to rule out inflammatory, infectious, auto-immune, vascular or neuropathic causes was initiated. An extensive laboratory test (ESR, CRP, ANA, ds-DNA AB, scleroderma AB, ENA AB, cardiolipin IGG/IGM, IGA, lysozyme, ACE, c-ANCA, p-ANCA, SS-B, SS-A) was all negative. In addition, neuroimaging was performed to rule out compressive lesions. MRI of the brain demonstrated small vessel atrophy. MRA brain demonstrated $40-50 \%$ stenosis of the left carotid artery. An MRI C-spine demonstrated normal spinal degenerative age-related changes. A temporal artery biopsy was performed and negative for pathologic changes consistent with giant cell arteritis. A supraorbital nerve biopsy was performed and demonstrated axonal degeneration with demyelination.

These findings were consistent with Facial Onset Sensory and Motor Neuropathy syndrome (FOSMN). The patient received amniotic membrane for treatment of neurotrophic keratopathy as well as medications for treatment of neuropathic pain. She started to lose weight and had weakness on her extremities. IVIG 40-gram dose of Octagam (approximately $1 \mathrm{~g} / \mathrm{kg}$ ) helped with generalized pain and weakness and she was able to walk again. Her vision loss stayed stable (Figure 1).

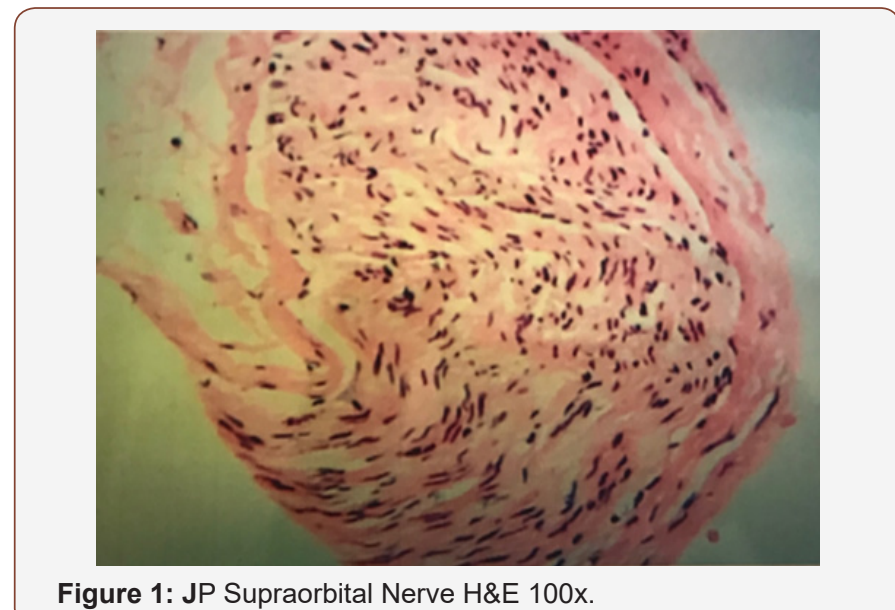

\section{Discussion}

FOSMN is a rare and slowly progressive motor neuron disorders.

A search in English articles from 2006 to 2018 showed that only 38 cases of FOSMN have been reported since it was first reported in 2006. FOSMN typically presents with slowly progressive numbness of the face followed by arms and neck weakness. Absent or reduced 
corneal and blink reflexes are the main pathognomonic ophthalmic presentation of this syndrome [1]. Vucic S [2] reported 4 patients diagnosed with FOSMN. The symptoms started with developed paresthesia and numbness in trigeminal nerve distribution, later involved the scalp, neck, upper trunk and upper limbs in sequential order. Motor involvement may cause cramps, fasciculations, dysphagia, and muscle weakness or atrophy. Neurologists and ophthalmologists should pay attention while visiting Patients presenting with bilateral trigeminal neuralgia associated with hypoesthesia of the face. One should consider to differentiate between trigeminal isolated sensory neuropathy (TISN), a benign and purely trigeminal neuropathy, and facial-onset sensory motor neuronopathy (FOSMN), a malignant life-threatening condition. No diagnostic criteria can yet differentiate the two conditions at their onset. Follow up on 13 patients with recent onset of trigeminal hypoesthesia and pain demonstrated the progression of five patients to FOSMN, while the other eight patients remained stable [3]. In both group of patients with TISN and FOSMN, neurological and histological examination documented a trigeminal nerve damage.

Although no clinical diagnostic criteria can distinguish TISN and FOSMN at onset, neurophysiological and nerve-biopsy findings show that in both diseases, trigeminal nerve damage affecting myelinated fibers and sparing unmyelinated fibers, which explain similar pathophysiological mechanisms in both. In 1959, Spillane and Wells reported 16 patients with a purely sensory, unilateral or bilateral trigeminal neuropathy [4]. later, Lecky and colleagues described 13 patients with idiopathic sensory disorders limited to the trigeminal region and referred to this condition as "idiopathic trigeminal sensory neuropathy" [5]. Recently, Vucic and colleagues indicated that in patients with bilateral trigeminal neuropathy a severe motor involvement may develop [2,6]. The treatment for this rare disease has stayed controversial. IVIG and plasmapheresis was recommended. We are reporting a rare case of FOSMN to increase neurologist and ophthalmologist's awareness and knowledge. Early diagnosis and prompt attention to wellbeing of cornea may prevent blindness from neurotrophic keratopathy. Clinical research over the pathophysiology and treatment of this rare but disabling disease may improve the patients' quality of life.

\section{Conclusion}

In patient with bilateral facial sensory motor neuropathy, ophthalmologist should consider FOSMN as one of the rare diseases that may cause this condition. Supraorbital nerve biopsy is necessary for diagnosis; demonstrating axonal degeneration with demyelination. Early treatment of neurotrophic keratopathy is essential. IVIG may help alleviating the generalized symptoms.

\section{Acknowledgment}

None.

\section{Conflicts of Interest}

No conflict of interest.

\section{References}

1. Zheng Q, Chu L,Tan L, Zhang H (2016) Facial onset sensory and motor neuronopathy. Neurol Sci 37(12): 1905-1909.

2. Vucic S, Tian D, Chong PS (2006) Facial onset sensory and motor neuronopathy (FOSMN syndrome): a novel syndrome in neurology. Brain 129(12): 3384-3390.

3. Cruccu G, Pennisi EM (2014) Trigeminal isolated sensory neuropathy (TISN) and FOSMN syndrome: despite a dissimilar disease course do they share common pathophysiological mechanisms. BMC Neurol 14: 248.

4. Spillane JD, Wells CE (1959) Isolated trigeminal neuropathy. A report of 16 cases. Brain 82: 391-416.

5. Lecky BR, Hughes RA, Murray NM (1987) Trigeminal sensory neuropathy. A study of 22 cases. Brain 110(6): 1463-1485.

6. Vucic S, Stein TD, Hedley Whyte (2012) ET FOSMN syndrome: novel insight into disease pathophysiology. Neurology 79(1): 73-79. 